\title{
Surfaces aren't superficial
}

\author{
The 2007 Nobel prizes in both physics and chemistry stem from investigations into the \\ properties of materials, but they share more in common than that.
}

At first glance, little might seem to connect the elucidation of reaction mechanisms on heterogeneous catalysts, for which Gerhard Ertl of the Fritz Haber Institute in Berlin was awarded last year's chemistry Nobel prize, and the discovery of giant magnetoresistance in magnetic multilayers, which earned the physics prize for Albert Fert of the Universite Paris-Sud and Peter Grünberg of the Forschungszentrum Jülich. But both awards point to the fundamental importance of surfaces and interfaces, and moreover both topics depend on a detailed understanding of the electronic structures of materials.

Ertl's award has brought a smile to the faces of everyone in the field, because he has for decades conducted diligent, undemonstrative work in an area that has never been particularly fashionable or celebrated. Yet the fact that it was only in the early 1980s that Ertl succeeded in constructing a complete picture of the basic steps in the catalytic conversion of nitrogen and hydrogen to ammonia on iron - the process, of inestimable industrial significance, that won Haber a Nobel in 1918 - shows just what an intellectually and experimentally demanding problem this was. Naturally, the question had received a lot of attention before then, but Ertl was able to use state-of-the-art experimental methods, particularly electron spectroscopies, to characterize all the steps that followed the rate-determining dissociation of adsorbed nitrogen.

Under Ertl's directorship, the Fritz Haber Institute became a place where researchers could deploy whatever approach was needed to attack tricky questions in surface chemistry: the philosophy was not to apply a particular technique to diverse problems, but to make the problem itself the focus, tackling it from all angles until an explanation

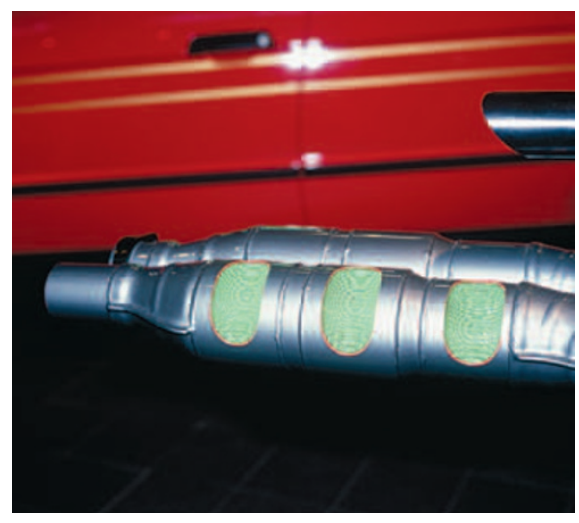

Catalytic converters are just one of the technologies that rely on a detailed understanding of surface catalysis.

emerged. In this way, Ertl played a central role in connecting bench-top science to industrial realities. Previously, it was unclear how relevant the idealized surface catalytic processes studied under high vacuum and on single crystals were to the operation of real catalysts. In recent years, such experimental approaches have been increasingly supplemented with sophisticated quantum-chemical calculations that can probe the fine details of how changes in a catalyst's surface electronic states influence the chemical processes that happen there.

Heterogeneous catalysis has always been a field led by pragmatic empiricism, so that processes such as Haber's, or more recently the use of catalytic converters in vehicle exhausts, can become pervasive technologies well in advance of a thorough understanding of how they work. Giant magnetoresistance (GMR) is also now the basis of an immense industry - hence the headlines describing the Nobel work as "the science behind the iPod". But what is striking here is the rapid timescale involved, a testament to the hunger of the information-technology companies for better ways of storing and reading digital data. Fert and Grünberg discovered only at the end of the 1980s that magnetic multilayers composed of films just a few tens of nanometres thick, in which magnetic and non-magnetic metals alternate, can generate very large changes in resistance in response to a magnetic field. Within less than a decade - thanks in considerable measure to the efforts of Stuart Parkin and his co-workers at IBM's Almaden research centre in California commercial GMR heads were reading out data from magnetic recording media.

Arguably this was one of the first real applications of nanotechnology. It was also the beginning of the field now known as spintronics, in which the quantummechanical spin of mobile electrons in conducting materials is used as a degree of freedom for information technology ${ }^{1}$. Spin-dependent electron scattering at the interface of the layers as their magnetization is switched creates resistance changes of not just a few percent, as in previous magnetoresistive materials, but an order of magnitude larger.

Fert continues to look for new materials physics that might have useful applications in data storage - as, for example, in his recent report of oxide thin films with both ferromagnetic and ferroelectric properties, which could permit four-state logic with non-destructive readout ${ }^{2}$. Whether or not such investigations pay off, it seems clear that we have by no means yet exhausted the diversity of interactions between electronic and magnetic states in matter, or ways to exploit them.

\footnotetext{
References

1. Chappert, C., Fert, A. \& Nguyen Van Dau, F. Nature Mater. 6, 813-823 (2007).

2. Gajek. M. et al. Nature Mater. 6, 296-302 (2007)
} 